\title{
Partizipative Führung: Auf den Spuren eines Konzeptes
}

\author{
Irma Rybnikova ${ }^{1}$ (D) Rainhart Lang ${ }^{2}$
}

Online publiziert: 15. April 2020

(C) Der/die Autor(en) 2020

\section{Zusammenfassung}

Dieser Beitrag der Zeitschrift Gruppe. Interaktion. Organisation. (GIO) befasst sich mit ausgewählten Konzepten zur partizipativen Führung, mit denen die Führungsforschung aufwartet. Das Ziel besteht darin, eine kritische Inspektion der den Konzepten zugrundeliegenden Annahmen über die Partizipation im Führungsgeschehen vorzunehmen. Dabei richten wir unseren Fokus zuerst auf die historischen Vorläufer der Führungspartizipation, wie die Überlegungen von Mary Parker Follett, Kurt Lewin, Robert Tannenbaum und Warren H. Schmidt. Anschließend diskutieren wir eine Reihe ausgewählter aktueller Konzepte aus dem Bereich partizipativer Führung in Bezug auf deren analytischen Kerndimensionen und rhetorischen Ansprüche. Die Konzepte durchleuchten wir in Bezug darauf, inwiefern diese auf die humanistisch inspirierte Demokratisierung von Organisationen mittels partizipativer Führung abstellen, oder partizipative Führung als ein weiteres Mittel zur Leistungssteigerung in Organisationen thematisieren, oder womöglich beides. Zum Schluss gehen wir darauf ein, welche dieser vielfältigen konzeptionellen Vorüberlegungen Eingang in die Diskussion zum agilen Management gefunden haben.

Schlüsselwörter Partizipative Führung · Agiles Management

\section{Participative leadership: in search of a (shifting) concept}

\begin{abstract}
This contribution to the Gruppe. Interaktion. Organisation (GIO) deals with selected concepts of participative leadership. The aim is to carry out a critical overview of the underlying assumptions about participation in leadership. We will first focus on the historical precursors of participative leadership, such as the reflections provided by Mary Parker Follett, Kurt Lewin or Robert Tannenbaum and Warren H. Schmidt. We then discuss a number of recent concepts from the field of participative leadership with regard to their analytical core dimensions and rhetorical claims. By outlining the conceptual landscape of participative leadership research, we examine whether the concepts focus on the humanistic inspired ideas of democratization in organizations by means of participation, or rather consider participative leadership as a just another means of improving performance in organizations, or possibly both. Finally, we discuss which of these various conceptual considerations have found their way into the debate on agile management.
\end{abstract}

Keywords Participative leadership - Agile management

Irma Rybnikova

Irma.Rybnikova@hshl.de

Rainhart Lang

r.lang@wirtschaft.tu-chemnitz.de
Department 2 Lippstadt, Hochschule Hamm-Lippstadt, Marker Allee 76-78, 59063 Hamm, Deutschland

2 Fakultät für Wirtschaftswissenschaften, Technische Universität Chemnitz, Thüringer Weg 7, 09126 Chemnitz, Deutschland 


\section{Partizipative Führung - ein Führungsmantra der Gegenwart?}

Gegenwärtige Führungskräfte beschreiben sich überaus gern als partizipativ Führende. Auch die Diskussion um das agile Management und agile Unternehmen trägt zur Popularisierung der partizipativen Führung bei, beruhen doch agile Managementprinzipien auf Selbstorganisation und Führungsdelegation (Gloger und Rösner 2014). Die Apologetinnen und Apologeten des agilen Managements bedienen sich daher sehr gern aus der Kammer der Führungslehre zu partizipativer Führung. In dieser Ideenkammer findet man aber allerlei Verschiedenes. Die konzeptionellen Analysen zur partizipativen Führung sind nicht nur hochgradig fragmentiert, sondern bisher auch nur unzureichend aufgearbeitet und zusammengefasst. Explizite Ausführungen zu partizipativer Führung sind in den landläufigen Lehrbüchern zum Thema rar und knapp gehalten, wie z. B. bei Northouse (2019), Weibler (2016), Gill (2011) oder Neuberger (2002). Eine Ausnahme bildet Yukl (2006, 2013, S. 105-134) mit einem Kapitel zu partizipativer Führung und Empowerment, ohne allerdings über die verhaltensorientierten Ansätze hinauszugehen (Lang 2014, S. 77 f.).

Vor allem vermisst man Übersichten aus kritischer Perspektive, die den Konzepten zugrunde gelegte Annahmen inspizieren. Diesen aus unserer Sicht defizitären Zustand möchten wir in unserem Beitrag angehen und eine konzeptionelle Verortung der Forschungslandschaft zu partizipativer Führung vornehmen. Es handelt sich um eine Forschungsübersicht, bei der die Frage beantwortet werden soll, inwiefern in den konzeptionellen Entwicklungen zum Thema partizipative Führung diese als eine weitere Möglichkeit zur Effizienzsteigerung in Unternehmen dargestellt wird oder stattdessen das emanzipativ-demokratische Versprechen, Führung als eine demokratiegerichtete Institution aufzufassen, eingelöst wird. Was dieser Beitrag hingegen nicht liefert, ist eine Definition des Partizipationsbegriffs. Indem wir verschiedene Verständnisse der partizipativen Führung aus den vorliegenden Konzepten herausschälen, wollen wir uns ganz bewusst dabei zurückhalten, diesen Konzepten eine eigene Vordefinition der Partizipation aufzubürden.

Das Hauptaugenmerk des Vorhabens liegt auf einer Analyse der klassischen und der aktuelleren - seit 1980 erschienener - Konzepte, die jeweils verschiedene Aspekte der Partizipation in Führungsprozessen thematisieren. Unser Fokus richtet sich jedoch an die Arbeiten, die Führung von Mitarbeiterinnen und Mitarbeitern in seiner Gesamtheit adressieren, nicht aber deren Teilaspekte oder Einzelinstrumente. Aufgrund des gelegten Fokus haben wir in unserer Auswahl einige wichtige benachbarte Forschungsstränge nicht einbeziehen können. Dazu gehört beispielsweise die arbeitspsychologische Forschung zur organisationalen Demokratie (z. B. Wegge et al. 2010; Weber et al. 2019), in der partizipative oder geteilte Führung eine prominente Rolle spielt, ohne allerdings das Verständnis der partizipativen Führung zu diskutieren, denn hauptsächlich konzentriert sich diese Literatur auf den Folgen der partizipativen Führung. Auch die Forschung zur partizipativen Zielsetzung, die mit intensiver und einschlägiger Literaturdebatte aufwartet (z. B. Wegge und Haslam 2005; Haslam et al. 2009; Kleigeld et al. 2011), wird in der vorliegenden Analyse nicht berücksichtigt, weil hier mit der Gestaltung der Zielvereinbarungen nur ein Teilaspekt der Führungspartizipation im Fokus steht.

Dabei spannen wir ein weites Spektrum auf, welches mit manch inzwischen in Vergessenheit geratenen Modellen anfängt, wie den Überlegungen von Mary Parker Follett oder der kooperativen Führung nach Wunderer und Grundwald (1980), und bis zu den aktuellen führungskritischen Konzepten reicht, wie Führung nach Bedarf von Blom und Alvesson (2014). Bei der Auswahl der zu diskutierenden Konzepte haben wir uns einerseits nach der konzeptionellen Dichte der in den Konzepten vorgebrachten Argumente gerichtet und andererseits nach der Popularität der Theorien in der aktuellen Führungsforschung. Das Ergebnis umfasst daher zum einen in konzeptioneller Hinsicht innovative Ansätze, die inzwischen in Vergessenheit geraten sind und/oder aktuell wenig Beachtung erfahren, wie die Ideen von Mary Parker Follett (1995), Gastill (1994), Wunderer und Grunwald (1980) oder Blom und Alvesson (2014). Andererseits sind dabei auch Theorien, wie demokratischer Führungsstil (Lewin et al. 1939) oder geteilte Führung, die sich seit Jahren einer ungebrochenen Aufmerksamkeit in den empirischen Studien zu Mitarbeiterführung erfreuen.

Unsere Analyse konzentriert sich auf das grundsätzliche Verständnis von partizipativer Führung im jeweiligen Ansatz sowie die thematisierten Ziele und Wirkungen. Wir wollen dabei insbesondere den rhetorischen Anspruch der Ansätze auf Lieferung einer Alternative zu klassischer Führung überprüfen, und zwar in Bezug darauf, ob der jeweilige Ansatz in seiner Begründung eine Demokratiorientierung (d.h. egalitäre und nicht zentralisierte Machtverteilung als Selbstzweck) oder eine Effizienzorientierung (d.h. egalitäre und nicht zentralisierte Machtverteilung als Mittel zur Effizienzsteigerung) aufweist. Die Demokratie- und die Effizienzorientierungen sind ausschließlich als eine analytische Trennung zu verstehen, deren Zweck darin besteht, die Unterschiede zwischen den Konzepten der partizipativen Führung aufzuspüren. Auch wenn der Gegensatz zwischen den beiden Dimensionen nicht notwendigerweise gegeben ist, ist er aus unserer Sicht zumindest informativ und ermöglicht eine kritische Beleuchtung der ideologischen Hintergründe, die in die Verständnisse für partizipative Führung eingelassen sind. Praktische Implikationen unserer Analyse 
zeigen wir auf, indem wir den konzeptionellen Zusammenhang zwischen den Konzepten partizipativer Führung und dem agilen Management untersuchen.

\section{Vorläuferkonzepte: Von Mary Parker Follett bis zum Führungsstilkontinuum nach Tannenbaum und Schmidt}

Eine der ersten Managementautorinnen, die sich stark mit Partizipation in Organisationen befasste, ist die Amerikanerin Mary Parker Follett. Von Peter Drucker zur Prophetin des Managementdenkens gekürt, ist sie in der gegenwärtigen Managementforschung gleichzeitig nahezu vergessen, sieht man von gelegentlichen Publikationen ab (z. B. McLarney und Rhyno 1999; Mendenhall und Marsh 2010). Follett liefert in ihren Werken zahlreiche Überlegungen, die partizipative Führung in Organisationen begründen, wobei ihre praktische Arbeit im sozialen Bereich die wesentliche Inspirationsquelle ihrer konzeptionellen Annahmen zur Führung ist.

Follett kritisiert die üblicherweise enge Verbindung zwischen Führung und der Person einer Führungskraft, die in der traditionellen Führungsforschung bis dato überwiegt, als einen kardinalen Fehler (Follett 1995, S. 168). Eine scharfe Kritik seitens der Autorin erfahren auch die autoritären Führungsstrukturen in Organisationen, die Potenziale von Gruppen unterminieren (Follett 1995, S. 168). Stattdessen plädiert Follett zu einer Re-Fokussierung der Führung auf die Situation und auf die Geführten. Die Autorin unterscheidet drei Führungsarten: positionsbezogene, persönlichkeitsbezogene und funktionsbezogene Führung (Follett 1995, S. 174) und sieht die funktionsbezogene Führung, der eine Entpersonalisierung von Anweisungen (Follett 1940b, S. 58) zugrunde liegt, als besonders geeignet an. Als Reaktion auf Sachzwänge sollen die Anweisungen aus den Anforderungen einer konkreten Situation abgeleitet werden.

Laut Follett ist Führung in Organisationen als ein Prozess zu sehen, bei dem „Führende und Geführte einem unsichtbaren Führer - dem gemeinsamen Ziel“ folgen (Follett 1995, S. 172). Es führt ein Ziel, keine Person. Die herkömmlichen Führungskräfte sind dann erfolgreich, wenn sie in der Lage sind, dieses Ziel deutlich zu machen. Diese unsichtbare, weil entpersonalisierte Führung ist zugleich eine multiple, weil geteilte und auf Kooperation aller Beteiligten beruhende Führung. Sie basiert auf einer ko-aktiven Macht und einer geteilten Verantwortung aller Mitglieder.

Die Geführten sind nicht nur als zu führende Elemente anzusehen, sondern als Ko-Akteurinnen und Ko-Akteure und aktive Mitgestaltende der Führung (Follett 1995, S. 170). Diese Zuwendung zu den Geführten bleibt nicht ohne Konsequenz für die Machtverhältnisse in einer Organisation. So ist Follett zufolge in Organisationen auf die
„Macht über“ bzw. die Dominanz über die Mitglieder zu verzichten und stattdessen die "Macht mit“ den Mitgliedern zu leben (Follett 1940a, S. 101; Child 2013, S. 79).

Ungeachtet ihrer für damalige wie heutige Zeit hochbrisanten Ideen zu Führung blieb Follett in der bisherigen Führungsforschung nahezu unbemerkt. Im Bereich partizipative Führung kann Follett jedoch zweifelsohne als eine Pionierin angesehen werden, weil sie nicht nur die autoritären Führungsstrukturen kritisiert und die Bedeutung der Geführten hervorhebt, sondern auch mit der funktionsorientierten Führung eine konzeptionell begründete Alternative zu traditionellen Führungsmodellen bietet.

Die Hinwendung zu einer humanorientierten, die Interessen der Mitarbeiterinnen und Mitarbeiter zumindest beachtenden Führung findet sich bereits in der als Human Relations bezeichneten Richtung der Managementtheorie und -forschung zu Beginn der 30er Jahre. Die durch George Elton Mayo und Fritz Roethlisberger geleiteten Forschungen zur humaneren Gestaltung der Arbeitsbedingungen in der Western Electric Company schlossen zunehmend auch Fragen der sozialen Beziehungen zwischen Führung und Mitarbeiterinnen und Mitarbeitern sowie innerhalb der Arbeitsgruppen ein. Der Grund dafür war, dass Führung als ein wichtiger Faktor für das Leistungsverhalten der Arbeiterinnen und die Arbeitsergebnisse der Gruppe erkannt wurde (vgl. Roethlisberger und Dickson 1939; Gesigora 2015; Steffensen 2015).

Die Autoren der Hawthorn-Studien sehen eine Partizipation vor allem im Sinne einer offenen Meinungsäußerung von Mitarbeiterinnen und Mitarbeitern und ihrer Beachtung durch das Management. Als Basis dafür werden die Beziehungsgestaltung (,freundlich“) und ein nicht-direktives, erkundendes Verhalten der Vorgesetzten in Interviewsituationen gesehen. Eine weitergehende Einbeziehung der Mitarbeiterinnen und Mitarbeiter, etwa in Entscheidungsprozesse wird dagegen nicht thematisiert.

Hinsichtlich der Einordnung der Führungsvorstellung ist zunächst auf den expliziten Fokus der Hawthorn-Studien auf Faktoren der Leistungs- und Effizienzsteigerung zu verweisen. Dies gilt auch für die ,sozialen“ Faktoren wie den Umgang der Führung mit den Mitarbeiterinnen und Mitarbeitern. Immer wieder verweisen die Autoren darauf, dass eine Nichtbeachtung der Mitarbeiterinteressen und -gefühle (,sentiments") negative Folgen für die Leistung hat, und umgekehrt eine entsprechende Beachtung positive Effekte zeitigt. Zugleich wird jedoch auch der soziale Charakter von Organisationen und die Beachtung und Erhöhung der Arbeitszufriedenheit als zusätzliches Ziel des Managementhandelns hervorgehoben (Steffensen 2015, S. 581).

Kritiker sprechen dagegen von einer Fortsetzung des Taylorismus mit anderen Mitteln, Soziotechnik, oder „CowManagement", alles Begriffe, die in den Human Relations, und damit auch der dort vorgeschlagenen Führung eine 
deutliche Mittel-Zweck-Beziehung sehen (vgl. Braverman 1974; Hinrichs 1984; Breisig 1990; Neuberger 2002; Steffensen 2015, S. 582; Kieser 2014). Die Kritiker weisen darauf hin, dass die ,freundliche“ Führung durchaus ins Gegenteil umschlagen konnte, und vor allem für Mitarbeiterinnen und Mitarbeiter galt, die sich gegenüber dem Management ,kooperationsbereit“" verhielten (vgl. Carey 1967; Kieser 2014).

Kurt Lewin gilt als einer der Stammväter der Sozialund Organisationspsychologie. Vor allem mit den in den 30er Jahren durchgeführten experimentellen Studien in Kleingruppen thematisierte er den Einfluss von Führungsund Erziehungsstilen auf das Verhalten und die Leistung von Gruppen (Lewin et al. 1939).

In dem Grundsatzartikel definieren die Verfasser zunächst verschiedene Arbeitskulturen, die sie als ,group atmospheres" bzw. "social or leadership climates" bezeichnen. Eine relevante Forschungsfrage ist dabei, inwieweit ein demokratisches Gruppenleben zwar angenehmer, ein autoritäres dagegen effizienter ist (Lewin et al. 1939, S. 271), aber auch, unter welchen Bedingungen sich vermehrt Verhaltensweisen wie Rebellion gegen Autoritäten, apathische Unterordnung, Suche nach Schuldigen, autoritäre Dominanz oder Attacken gegen Außenseiterinnen und Außenseiter zeigen. In den Experimenten mit Schulkindern übernahmen Lehrerinnen und Lehrer die Rollen der Führenden, und traten entsprechend den festgelegten Verhaltensmustern autoritär, demokratisch oder nicht (en. laissez-faire climate) auf. Das demokratische Muster war durch folgende Aktivitäten der Führungsperson geprägt (Lewin et al. 1939, S. 273):

- Alle wichtigen Aktivitäten waren Gegenstand einer Diskussion und Entscheidung in der Gruppe, assistiert und ermutigt durch die Führende oder den Führenden.

- Die künftigen Aktivitäten und Schritte hin zu einem Gruppenziel wurden in der ersten Diskussion festgelegt. Die oder der Führende gab technische Unterstützung und machte ggf. mehrere Vorschläge für Entscheidungen.

- Die Gruppenmitglieder waren frei in der Entscheidung, mit wem sie zusammenarbeiten wollten, und die Aufgabenverteilung wurde der Gruppe überlassen.

- Die oder der Führende war möglichst objektiv und faktenbasiert bei Lob und Kritik, und verhielt sich wie ein normales Gruppenmitglied, ohne zu viel Arbeit zu übernehmen.

Die Effekte der unterschiedlichen Führungsumgebungen wurden durch Beobachtungen, Interviews und verschiedene Testverfahren sowie Einschätzungen der Zielerfüllung ermittelt, wobei die Autoren zwischen individuellen und gruppenzentrierten Ergebnissen unterschieden (Lewin et al. 1939, S. 276 ff.). Während bei der Gruppe mit einem autoritären Führungsmuster im ersten Experiment deutlich häufi- ger aggressives und feindseliges Verhalten gefunden wurde als in der Gruppe mit demokratischem Muster, wurden im zweiten, weitaus differenzierteren Experiment, inklusive einer Laissez-faire-Umgebung, sehr unterschiedliche Befunde ermittelt. Aggressionen und feindseliges Verhalten traten häufig auch in Umbruchsituationen auf, wenn die Führungssituationen etwa von autokratischer zu demokratischer oder Laissez-faire-Umgebung oder umgekehrt wechselte. Auch wenn die Autoren einen deutlichen Einfluss der durch die Führung geschaffenen Kulturmuster sehen, so warnen sie angesichts der differenzierten Befunde vor einer Generalisierung.

Die Studie von Lewin und Kollegen wird häufig als Startpunkt der Führungsstilforschung gesehen. Gerade durch die Kontrastierung von Autokratie und Demokratie wird ein Feld aufgespannt, das die Führungs(stil)forschung lang und nachhaltig dominiert (hat).

Bei der Bewertung des Beitrags von Lewin und Kollegen für Überlegungen einer partizipativen Führung ist zu beachten, dass das Forschungsinteresse und der Fokus, auch vor dem historischen und biografischen Hintergrund Lewins, primär auf den Wirkungen autoritärer Systeme und Führungsmuster und ihren Folgen für ein als negativ oder deviant charakterisiertes Verhalten von Gruppen und Individuen liegt. Demokratie und Laissez-faire dienen als Kontrastfolien in positiver wie negativer Hinsicht. Auch sie werden primär bezüglich der o.g. negativen Verhaltensweisen untersucht. Die Zielerreichung spielt dagegen eine untergeordnete Rolle. Allerdings gehen die Autoren davon aus, dass durch ein relativ stabiles Verhaltensmuster einer zentralen Führungsperson ein entsprechendes Führungsklima entsteht, das auf das Verhalten der beteiligten Akteurinnen und Akteure einwirkt. So ist die demokratische Führungsumgebung idealtypisch als weitestgehende Partizipation beschrieben, aber eben nur wenig ausgearbeitet.

Robert Tannenbaum und Warren H. Schmidt (1973) haben mit ihrem Konzept einen weiteren „Blockbuster“ für Managementlehrbücher geliefert, welcher in der Diskussion um partizipative Führung als ein klassisches Modell gilt. Bei einem genaueren Blick in den Originaltext stellt es sich jedoch heraus, dass mit ihrem Führungskontinuum die beiden Autoren keine ausführliche Betrachtung der partizipativen Führung liefern wollten. Vielmehr ging es ihnen darum, die Gegenüberstellung zwischen dem autoritären und demokratischen Führungsstil aufzubrechen und eine Vielfalt möglicher Führungsweisen aufzuzeigen sowie eine praktische Entscheidungshilfe für die Führungskräfte in Unternehmen anzubieten. Die zur damaligen Zeit stark ideologisch aufgeladene Diskussion zwischen dem demokratischen (,schwachen“) und dem autoritären (,starken“) Führungsverhalten verunsichere die Führungskräfte, so das Argument der beiden Autoren, weil ausschließlich demokratische Führung als gesellschaftlich akzeptabel dargestellt 
wird, wohingegen die starke Führung diskreditiert wird. Zudem würde diese Denke die große Breite der Führungsoptionen ausblenden. Man kann sich schwer des Eindrucks verwehren, dass Tannenbaum und Schmidt damit die ,,sozialen Laborexperimente" in Anleitung von Kurt Lewin kritisieren.

Als einen Gegenentwurf zu den beiden Führungsstilen entwickeln Tannenbaum und Schmidt ein Führungskontinuum, welches sich zwischen der Führungsorientierung und der Gefolgschaftsorientierung erstreckt und insgesamt sieben Formate des Führungsverhaltens umfasst. Auf der führungsorientierten Seite verorten die Autoren die bestimmenden Verhaltensweisen der Führungskräfte, wie „Führungskraft trifft Entscheidung und gibt sie bekannt" oder „Führungskraft verkauft seine Entscheidung“. Im Mittelfeld des Kontinuums schwächt sich die Bestimmtheit durch Führungskraft ab und die Ausrichtung auf die Geführten-Gruppe wird deutlicher: ,Führungskraft stellt Ideen vor und lädt zu Fragen ein“, „Führungskraft stellt vorläufige Entscheidung als veränderbar vor". Anschließend geht das Kontinuum in eine aktive Einbindung der Untergebenen bei der Entscheidungsfindung über, weswegen die Freiheitsgrade der Beschäftigten zunehmen: „Führungskraft stellt das Problem dar, erhält Vorschläge und trifft Entscheidung“, „Führungskraft gibt Grenzen vor und überlässt die Entscheidung der Arbeitsgruppe“. Das letzte und damit das am stärksten an Untergebenen orientierte Führungsformat aus Sicht von Tannenbaum und Schmidt ist es, wenn Führungskraft „,der Arbeitsgruppe es erlaubt, innerhalb der durch Vorgesetzten definierten Grenzen frei zu agieren" (Tannenbaum und Schmidt 1973, S. 4). Das sei zugleich ein ,extremer Grad der Gruppenfreiheit“, die in Organisationen äußerst selten vorkomme (Tannenbaum und Schmidt 1973, S. 5).

Im Führungskontinuum ist die Idee der partizipativen Führung zwar eingelassen, explizit wird sie aber nicht diskutiert. Die Autoren verbinden mit partizipativer Führung die von einer Führungskraft eingeräumte Möglichkeit einer Arbeitsgruppe zur Mitwirkung bei der Entscheidungsfindung. Im Originaltext fokussieren sich Tannenbaum und Schmidt ausschließlich auf die Beschreibung verschiedener Grade der Einbeziehung der Untergebenen. Weder verwenden sie den Begriff der partizipativen Führung, noch setzen sie es mit einem von ihnen beschriebenen Führungsverhalten gleich. Stattdessen befassen sich die beiden Autoren mit den Rahmenbedingungen, die das jeweilige Führungsformat mehr oder weniger geeignet erscheinen lassen, wie z.B. das Wertesystem der Führungskräfte, die Bedürfnisse und Neigungen der Untergebenen, oder die Gegebenheiten der Situation. So empfiehlt sich laut Tannenbaum und Schmidt eher die alleinige Entscheidung durch Führungskraft, wenn diese nur schwer die Unsicherheit tolerieren kann, die Untergebenen mangelnde Kompetenz aufweisen und Zeitdruck vorherrscht.
Erst in den deutschen Übersetzungen des Konzepts finden sich Zuordnungen zur partizipativen Führung statt. So lesen wir beispielsweise bei Dirk Holtbrügge, dass als ,,partizipativ“ nicht die letzte Ausprägung des Führungsstilkontinuums bezeichnet wird - diese heißt hier ,delegativ“ -, sondern die drittletzte, und zwar wenn die Führungskraft Problem präsentiert, um Vorschläge seitens der Untergebenen bittet und auf dieser Basis alleine die Entscheidung trifft (Holtbrügge 2018, S. 238). Die ursprüngliche Idee der Partizipation wird auf diese Weise erheblich modifiziert: anstatt eines Kontinuums mit zahlreichen möglichen Gradierungen wird partizipative Führung mit einer der Zwischenstufen gleichgesetzt. Damit findet eine Einengung und Vereindeutigung statt, die weder dem Originalkonzept von Tannenbaum und Schmidt noch der praktischen Vielfarbigkeit der Partizipation bei Entscheidungsfindung gerecht wird.

Das gerade in der Betriebswirtschaftslehre weit verbreitete normative Entscheidungsmodell nach Vroom und Yetton (1973) sowie Vroom und Jago (1978) adressiert explizit unterschiedliche Grade der Partizipation von Mitarbeiterinnen und Mitarbeitern im Entscheidungsprozess. In Abhängigkeit von der jeweiligen Entscheidungssituation soll das Modell helfen, das jeweils effektivste Entscheidungsverfahren auszuwählen. Vroom und Yetton geben fünf verschiedene ausgewählte Entscheidungsvarianten an: autoritäre Alleinentscheidung, autoritäre Entscheidung nach Informationen durch Mitarbeiterinnen oder Mitarbeiter, konsultative Entscheidung nach Einzelberatung mit Mitarbeiterinnen und Mitarbeitern, konsultative Entscheidung nach Gruppenbesprechung sowie Problemlösung und Entscheidung durch die Gruppe (Vroom und Yetton 1973, S. 13). Die Kernannahme des Modells ist dabei, dass steigende Partizipation auch jeweils die Akzeptanz von Entscheidungen durch die Mitarbeiterinnen und Mitarbeiter steigert, und ein stärkerer Einfluss bei Entscheidungsprozessen auch deren Motivation erhöht, die Entscheidungen engagiert umzusetzen (vgl. Yukl 2013, S. 112).

Die Autoren geben dazu verschiedene situative Kontextfaktoren an, die die Auswahl der effektiven Entscheidung bestimmen: Strukturiertheit des Entscheidungsproblems, Bedeutung der Qualität der Problemlösung, Verfügbarkeit von ausreichenden Informationen, Bedeutung der Akzeptanz der Entscheidung, Akzeptanz von Einzelentscheidungen, Akzeptanz der Organisationsziele durch die Mitarbeiter sowie Konfliktwahrscheinlichkeit. In einem erweiterten Modell (Vroom und Jago 1978) wurden noch Zeitdruck und Entwicklung(sstand) der Mitarbeiterinnen und Mitarbeiter als Faktoren einbezogen. Für die Zuordnung werden schließlich Regeln aufgestellt, die neben der Auswertung sozialpsychologischen Studien (vgl. Neuberger 2002, S. 504 f.), überwiegend auf Plausibilitätsüberlegungen der Autoren beruhen, etwa: 
- wenn die Entscheidungsqualität wichtig ist, der/die Vorgesetzte aber nicht über alle notwendigen Informationen verfügt, ist die autoritäre Einzelentscheidung auszuschließen (Informationsregel) oder

- wenn die Entscheidungsqualität wichtig ist, die Mitarbeiterinnen und Mitarbeiter aber die Organisationsziele nicht teilen, ist die Problemlösung durch die Gruppe auszuschließen (Zielübereinstimmungsregel).

Wenn in bestimmten Situationen mehrere Stile in Frage kommen, wird bei hoher Bedeutung von Entscheidungsqualität und Zeitdruck eher zu autoritären Entscheidungen geraten; ist dagegen die Akzeptanz wichtig, sollte in solchen Situationen partizipativen Stilen der Vorzug gegeben werden.

Die Vorstellung von partizipativer Führung reduziert sich dabei zunächst auf einen spezifischen Stil des Entscheidungsverhaltens von Führungskräften. Das Modell stellt eine Kombination eindimensionaler Führungsmodelle (s. Tannenbaum und Schmidt oben) und klassischer Kontingenzmodelle der Führung (z.B. Fiedler 1967) dar. Die Partizipation der Mitarbeiterinnen und Mitarbeiter wird dann als sinnvoll und effektiv angesehen, wenn ihr Wissen und Motivation zum Treffen und zur Umsetzung der Entscheidung benötigt werden. Es geht also um von der Führungskraft gewährte, in deren Effizienz- und Machtkalkül liegende, ,,dosierte“ Mitwirkung an Entscheidungen, da über den Zeitdruck jederzeit eine autoritäre Entscheidung zu rechtfertigen ist. Ziele des Führungshandelns bleiben unbeachtet (vgl. Neuberger 2002, S. 509). Machtkritische und weitergehende demokratische Begründungen spielen keine Rolle.

\section{Gegenwärtige Konzepte}

Aus der inzwischen weit verzweigten Landschaft der aktuellen Konzepte, die mit Partizipation und Führung befassen, stellen wir im Folgenden einige ausgewählte ausführlicher dar. Dabei konzentrieren wir uns einerseits auf Überlegungen, die weniger stark beachtet werden, aber relevante Entwicklungen darstellen, andererseits auf die prominenteren und oft rezipierten Ansätze. Zu Beginn werden wir somit weniger beachtete Ansätze entsprechend ihrer Erscheinungschronologie vorstellen, und zwar jene von Wunderer und Grundwald (1980), Gastil (1994), Raelin (2006) sowie Blom und Alvesson (2014). Zwar wird in diesen Ansätzen mit verschiedener Semantik operiert, in allen wird jedoch auf jeweils eigene Weise die Idee der Führungsteilung und -partizipation vertieft. Auf diese Art verdeutlichen wir die Vielfalt der Konzepte zur partizipativen Führung, bevor wir auf aktuell stark rezipierte und von einem breiten Kreis an Autorinnen und Autoren beforschte Ansätze der geteilten und der verteilten Führung eingehen, die häufig als ausschließliche Repräsentanten der partizipativen Führung gelten. Einige der besprochenen Ansätze sind stärker demokratieorientiert, indem sie partizipative Führung als Ausdruck demokratischer Werte in Unternehmen erachten, andere sind hingegen effizienzbezogen, sofern sie gestiegene Leistungseffizienz durch partizipative Führung in den Vordergrund stellen. Ohne einen Anspruch auf Vollständigkeit zu erheben, bezwecken wir mit der nachfolgenden Übersicht vielmehr eine Schlaglicht-Betrachtung, die verschiedene Verästelungen und Perspektivenvielfalt der aktuellen Diskussion um partizipative Führung erhellen soll.

\subsection{Kooperative Führung nach Wunderer und Grunwald}

Als wichtiges und frühes Beispiel im Kontext demokratieorientierter Konzepte partizipativer Führung im deutschsprachigen Raum ist die kooperative Führung nach Wunderer und Grunwald (1980) zu nennen. Das Interesse an einer verstärkten Kooperation und Partizipation in Unternehmen ist dabei vor dem Hintergrund der gesellschaftlichen Entwicklung in Deutschland in den späten 60er und 70er Jahren sowie der rechtlich verbindlichen Arbeitnehmermitbestimmung mit dem Betriebsverfassungsgesetz von 1972 zu sehen. Den kulturellen Hintergrund bildeten Prozesse des gesellschaftlichen Wertewandels mit einer zunehmenden Bedeutung emanzipatorischer Wertvorstellungen bei stark abnehmender Akzeptanz von Werten wie Gehorsam und Unterordnung, mit Folgen für die gesellschaftlichen Führungserwartungen. Auch der sich abzeichnende Wandel in der Technologie und den Arbeitsstrukturen spielten eine wichtige Rolle bei der Entstehung und Verbreitung des Konzeptes (vgl. Wunderer 1995, S. 1375).

Wunderer und Grunwald verstehen kooperative Führung als ,zielorientierte Einflussnahme zur Erfüllung gemeinsamer Aufgaben in/mit einer strukturierten Arbeitssituation unter wechselseitiger, tendenziell symmetrischer Einflussausübung und konsensfähiger Gestaltung der Arbeitsund Sozialbeziehungen“ (Wunderer und Grundwald 1980, S. 17).

Sie beziehen sich dabei auch auf die Arbeit zu Führungsformen von Seidel (1978), und arbeiten zwei Hauptdimensionen einer kooperativen Führung heraus (Wunderer und Grunwald 1980; Wunderer 1995, S. 1377 f.):

- die Beteiligung der Mitarbeiterinnen und Mitarbeiter am Entscheidungsprozess, die Teilhabe an der Führung (partizipative Dimension) und

- die partnerschaftlichen bzw. gruppenbezogenen interpersonellen Arbeits- und Führungsbeziehungen, die Teilnahme an der Führung (pro-soziale Dimension). 
Im Unterschied und in Abgrenzung zu den Arbeiten zum kooperativen oder partizipativen Führungsstil (vgl. Tannenbaum und Schmidt 1973; Vroom und Yetton 1973) betonen die Verfasser die besondere Bedeutung der pro-sozialen Dimension, die sie als wichtige und unverzichtbare Ergänzung zur Beteiligung am Entscheidungsprozess ansehen.

Die kooperative Führung wird als idealtypischer Zustand angesehen, bei dem beide Dimensionen eine hohe Ausprägung aufweisen. Kooperative Führung beruht dabei auf drei zentralen Werten, die die Akteurinnen und Akteure am Führungsprozess teilen müssen, und die zugleich in der Organisationskultur im Sinne einer Vertrauenskultur verankert sein sollen: Arbeit und Leistung, Wechselseitigkeit, Selbstverwirklichung. Zu Untersetzung entwickeln die Verfasser eine Anzahl von Merkmalen, die das Modell der kooperativen Führung charakterisieren (Wunderer und Grunwald 1980, S. 99f.), u.a. gemeinsame Einflussausübung durch Gruppenmitglieder, Sachautorität, bei der der jeweils fachlich kompetente den (Teil-)Prozess führt, multilaterale Informations- und Kommunikationsbeziehungen innerhalb der Gruppe, Konfliktregelung durch Aushandeln und Verhandeln, Gruppenorientierung statt individueller Orientierung in der Führung, Vertrauen statt Kontrolle als zentrales Steuerungsmedium, Bedürfnisbefriedigung der Mitarbeiterinnen und Mitarbeiter und Vorgesetzten sowie Ziel- und Leistungsorientierung.

Die veränderten gesellschaftlichen Rahmenbedingungen führen dazu, dass von der kooperativen Führung neben einer größeren gesellschaftlichen und Mitarbeiterakzeptanz aufgrund der motivationalen und legitimatorischen Wirkungen auch ein wesentlicher Leistungseffekt erwartet wird.

Die Verfasser sind sich durchaus bewusst, dass es sich um ein normatives Idealmodell handelt, dessen Umsetzung im Kontext der Machtverhältnisse vor allem in privatwirtschaftlichen Unternehmen nur schwer möglich ist. Wunderer konstatiert daher in den 90er Jahren einen Wandel von einem normativ-ethischen Idealstil kooperativer Führung hin $\mathrm{zu}$ einem praktisch-normativen Stil, den er als situativ-kooperative Führung bezeichnet (Wunderer 1995, S. 1380).

Die kurze Darstellung des Konzeptes der kooperativen Führung verdeutlicht, dass der Schwerpunkt des Konzeptes auf der Interaktion und der Führungsbeziehung liegt, die durch Zusammenarbeit und wechselseitigem Einfluss gekennzeichnet ist. Aspekte einer Verteilung der Führung auf Mitarbeiterinnen und Mitarbeiter (Sachorientierung) aber auch der gemeinschaftlichen Führung in und durch die Gruppe (gemeinsame Einflussausübung, Gruppenorientierung) markieren verschiedene Aspekte der Partizipation im Führungsprozess und erweitern den sehr engen Blick der klassischen Sicht auf partizipatives Führungsverhalten. Darüber hinaus wird auch das organisatorische (Vertrauenskultur) wie das gesellschaftliche Umfeld (gesellschaftliche
Führungserwartungen in Richtung Kooperation und Partizipation) thematisiert. Vor diesem Hintergrund ist $\mathrm{zu}$ konstatieren, dass die Demokratieorientierung im Konzept der partizipativen Führung klar dominiert. Es wird angenommen, dass der leistungsbezogene Führungserfolg auch und gerade durch Kooperation und Partizipation erreicht werden kann, weil so eine Übereinstimmung der praktizierten Führung mit den Mitarbeiterinteressen und den gesellschaftlichen Erwartungen erreicht werden kann. Diese Annahmen sind vor allem für Kulturen und Institutionensysteme charakteristisch, die für einen kooperativen Kapitalismus bzw. kooperative Geschäftssysteme stehen, ohne dass die Autoren diesen Bezug stärker thematisieren.

Bei den Grundannahmen fällt auf, dass die Integration von Demokratie- und Leistungsorientierung im Konzept als unproblematisch oder als gestaltbar angesehen wird. Widersprüche, zu erwartende Konflikte zwischen den verschiedenen Merkmalen (z.B. Ziel- und Leistungsorientierung vs. Bedürfnisbefriedigung der Mitarbeiter und Vorgesetzten) werden kaum diskutiert bzw. als Probleme der Implementierung deklariert. Vor diesem Hintergrund ist die Kritik verständlich, die kooperative Führung als Führungsideologie, Führungsmythos (Gemeinschaftsmythos: „Wir sitzen alle in einem Boot" nach Neuberger 2002, S. $58 \mathrm{ff} ., 104 \mathrm{f}$.) oder Managementmode bezeichnet.

Die Problematik der dem Konzept zugrunde liegenden Annahme einer Werte- und Interessenharmonie äußert sich auch in den massiven Umsetzungsproblemen. Wunderer selbst nennt u.a. narzisstische Persönlichkeitsstrukturen von Vorgesetzten, Tendenzen zur Verschleierung der Entscheidungen, technologische ,Sachzwänge“ und starre, oft rechtlich zementierte Verantwortungsstrukturen sowie die geringe Bereitschaft der Führungskräfte zur Abgabe und der Mitarbeiterinnen und Mitarbeiter zur Übernahme von Macht und Verantwortung (vgl. Wunderer 1995, S. $1371 \mathrm{ff}$.) als Ursachen.

\subsection{Demokratische Führung nach Gastil}

Ausgehend von der Ursprungsidee der demokratischen Führung nach Kurt Lewin, unterbreitet Gastil (1994) in seiner Veröffentlichung ein konkretisiertes Konzept der demokratischen Führung, das in unserer Sicht als ein weiteres Modell der demokratieorientierten partizipativen Führung anzusehen ist. Die demokratische Führung besteht dem Autor zufolge darin, das Verhalten von Menschen auf eine Art zu beeinflussen, die mit den demokratischen Prinzipien und Prozessen im Einklang steht und die demokratischen Prozesse unterstützt. Zu solchen demokratischen Prinzipien und Prozessen zählt der Autor die Selbstbestimmung, Inklusivität sowie Beteiligungsgleichheit. Der Autor hebt hervor, dass demokratische Führung sich durch drei Funktionen auszeichnet: 
- Aufteilung der Verantwortung unter allen Mitgliedern anstatt sie auf eine Person zu konzentrieren;

- Ermächtigung (en. empowerment) von Gruppenmitgliedern durch Mentoring und Unterrichten im kritischen Denken und Verantwortungsübernahme;

- Unterstützen von Entscheidungsprozessen in der Gruppe durch ein umsichtiges Berücksichtigen von Ideen, die die Mitglieder unterbreiten, emotional gesunde Rahmenbedingungen und Mitgliederbeziehungen in der Gruppe sowie das Aufrechterhalten offener Debatten (Gastil 1994, S. 958).

Je nach der Gruppensituation können die meisten Gruppenmitglieder, einige oder nur ausgewählte Mitglieder die Führungsfunktionen erfüllen. Dabei übernehmen sie abwechselnd die Rolle der Führungskraft oder die eines Mitglieds. Das Ergebnis der demokratischen Führung laut Gastil ist Pluralität und Vielfalt anstatt des Monopols der Führung. Die Ent-Heroisierung der Führung erfolgt in diesem Konzept dadurch, dass der analytische Blick sich den Geführten zuwendet. So ist laut Autor die demokratische Führung darin zu sehen, wenn sich jede oder jeder in der Gruppe ermächtigt fühlt, Projekte und Ideen zu initiieren oder zu stoppen oder andere herauszufordern. Keines der Gruppenmitglieder kann der Führung entkommen, weil jedes Gruppenmitglied irgendwann für eine begrenzte Zeit irgendeine der Führungsfunktionen übernimmt. Die temporäre Natur der Führungsaufgabe basiert darauf, dass alle Rollen, darunter auch jene der Führung, innerhalb einer Gruppe rotieren (Gastil 1994, S. 962).

Gastil thematisiert auch einige Voraussetzungen, der demokratischen Führung auf der Seite der Geführten und der Situationen. Demokratische Führung erfordert von den Mitgliedern einige besondere Fähigkeiten, die sie als „demokratische Geführten“ auszeichnen. Darunter zählt der Autor die Bereitschaft, Verantwortung zu übernehmen und nicht blind die von der Führungskraft gegebenen Rahmenbedingungen zu akzeptieren (Gastil 1994, S. 963). Im Gegenzug sind sie für ihre Entscheidungen und Tätigkeiten verantwortlich. Des Weiteren sind die Mitglieder in Bezug darauf reflektiert, wie sie die Führungsfunktionen ausfüllen können und streben nach Erweiterung ihrer Führungsfähigkeiten. Dieses Streben beruht auf dem Verständnis aller Bürgerinnen und Bürger als Individuen, die gleichermaßen in der Lage sind, zu führen wie zu folgen.

Neben den genannten Voraussetzungen an Akteure betrachtet Gastil auch, unter welchen Rahmenbedingungen demokratische Führung gedeihen kann. Die demokratische Führung ist aus seiner Sicht besonders dann geeignet, wenn Mitarbeiterinnen und Mitarbeiter sich eine solche Führung wünschen, erforderliche Kompetenzen besitzen oder die Anliegen mehrere Personen betreffen. Als ein Beispiel für demokratische Führung bringt Gastil politische Foren aus den USA an (en. National Issues Forums). Damit wird deutlich, dass es dem Autor um eine demokratische Führung allen voran in den politischen Kontexten geht.

\subsection{Kollaborierende Führung nach Raelin}

Einen der aktuelleren Ansätze in Verbindung mit partizipativen Führungsprozessen liefert der Amerikaner Joseph Raelin mit seinem Konzept der kollaborierenden Führung (en. collaborative leadership). In seinen seit zwei Jahrzehnten erscheinenden Veröffentlichungen zum Thema erfuhr die Idee der kollaborierenden Führung verschiedene Deutungen. Gleich geblieben ist das Hauptanliegen: die Kritik der individualistischen Perspektive auf Führung und der Vorschlag eines alternativen Paradigmas. Der Autor plädiert zur Abkehr von der dominierenden, ausschließlich auf die Führungsperson zentrierten Führungslehre und unterbreitet stattdessen eine Sicht, bei der Führung grundsätzlich als ein pluraler Prozess mit zahlreichen und wechselnden Beteiligten - Kollaborierende - aufzufassen ist.

Die Begründung dieser Führungsperspektive erfuhr bei Raelin eine bemerkenswerte Entwicklung. Während in den früheren Arbeiten eher ergebnis- und effizienzbezogene Argumentation anzutreffen war, knüpft der Autor in seinen letzten Arbeiten viel stärker an die analytisch anspruchsvollere und aus unserer Sicht stärker demokratiebezogene Praxis-Theorie an und verzichtet weitestgehend auf normative Argumente. So ist in einem der früheren Werke, nachzulesen, dass kollaborierende Führung auf dem einfachen, laut dem Autor ,humanistischen“, aber im Grunde rein utilitaristischem Prinzip beruht: „Wenn Menschen die Möglichkeit erhalten, sich in ihren Unternehmen zu beteiligen, ist deren Commitment gesichert" (Raelin 2006, S. 155).

Kollaborierende Führung nach Raelin basiert auf drei Prinzipien und vier operativen Perspektiven. $\mathrm{Zu}$ den drei Prinzipien gehört der urteilsfreie Dialog, die Fähigkeit, sich der kritischen Auseinandersetzung mit den Kolleginnen und Kollegen auszusetzen, und die Überzeugung, der Austausch könne einzigartige und neue Ergebnisse hervorbringen. Die vier operativen Perspektiven besagen, dass kollaborierende Führungskräfte gleichzeitig und kollektiv sowie gegenseitig und anteilnehmend sind. Gleichzeitig und kollektiv bedeutet, dass mehrere Führende in einem Projekt oder Arbeitsgruppe agieren und dadurch bereitwillig ihre Macht und Rechte untereinander teilen. Die Gegenseitigkeit und Anteilnahme beruhen auf der Würde eines jeden Individuums und Respekt jedem Beitrag gegenüber, unabhängig von der Herkunft oder hierarchischer Position der Beitragenden.

In seinen späteren Veröffentlichungen (z. B. Raelin 2014) unternimmt der Autor eine konzeptionelle Einbettung der kollaborierenden Führung in die Praxisperspektive. Noch entschiedener lehnt er dabei die heroisierende individualistische Perspektive auf Führungskräfte ab. Im Fokus der 
praxisorientierten kollaborierenden Führung steht „die Aktivität aller, die sich engagieren, deren soziale Interaktionen, Reflexionen und Anpassungen an die zu verrichtenden Aufgaben“ (Raelin 2014, S. 4). Die in den früheren Werken aufgeführten Prinzipien und Perspektiven der kollaborierenden Führung bleiben weiterhin gültig, lediglich werden sie nun als Eigenschaften der kollaborierenden Agenturhaftigkeit (en. collaborative agency) eingebunden. Die Führung wird dann als ein Prozess angesehen, in dem andauernde Interaktion der menschlichen und der nicht-menschlichen Akteure das Führen hervorbringt. Dabei zeichnet sich die kollaborierende Führung aus Sicht vom späteren Raelin zumindest durch drei ethische Prinzipien aus: 1) Demokratie und $\mathrm{Zu}$ gangsoffenheit zu Führung für alle Beteiligte aufgrund der individuellen Würde, 2) emanzipativer, beitragsfördernder Dialog und 3) Nachhaltigkeitsorientierung i.S. v. einer potenziellen langfristigen Umschichtung der Machtverteilung in Organisationen (Raelin 2014, S. 14 ff.). Somit legt Raelin mit der kollaborierenden Führung insofern ein bemerkenswertes Konzept, das zu Beginn noch stark effizienzbezogen begründet wurde, später aber eine ausschließlich demokratieorientierte Reformulierung erfuhr, ohne dass der inhaltliche Kern des Konzepts selbst abgeändert oder konkretisiert wurde.

\subsection{Führung nach Bedarf nach Blom und Alvesson}

Vor dem Hintergrund einer eher systemischen Betrachtung von Führung, bei der diese nur einer der möglichen Modi zur Steuerung von Organisationen (en. modes of organizing) ist (Alvesson und Blom 2018), haben sich Blom und Alvesson (2014) in einer qualitativen empirischen Studie mit der Frage beschäftigt, wie Geführte an der Führung mitwirken, indem sie die Führung durch formale Vorgesetzte (en. managerial leadership) beeinflussen, begrenzen oder initiieren.

Führung und Führungsbeziehungen zwischen den beteiligten Akteuren werden dabei als ko-konstruiertes, dynamisches Ergebnis eines kollektiven Prozesses der Interaktion, Meinungsbildung, und Aushandlung zwischen den beteiligten Akteuren, formalen und emergenten Führern, und Geführten, angesehen (Blom und Alvesson 2014, S. 345). Die Autoren schließen hier an die Literatur zur geteilten und verteilten Führung an, betonen aber besonders die aktive Einflussnahme der Geführten auf das Verhalten der formalen Führungskräfte, indem sie Führungsaktivitäten ein- und herausfordern (Blom und Alvesson 2014, S. 346f.) Sie bezeichnen diese Aktivitäten der Geführten als Führung nach Bedarf (en. leadership on demand). Geführte werden so zu (An-)Treibenden der Führung (Blom und Alvessson 2014, S. 353).

Mit Blick auf eine Partizipation im Führungsprozess handelt es sich bei dem Ansatz von Blom und Alvesson um eine echte weitgehende Beteiligung der Geführten. Die grundsätzlich kritische, emanzipatorische Perspektive der Autoren stellt sowohl Führung als zentralen organisationalen Steuerungsmechanismus wie auch die Führungskräfte als zentrale Akteurinnen und Akteure infrage. Jenseits der Kritik ist sie dabei weniger normativ, sondern versucht, eine dem Führungsgeschehen adäquatere Beschreibung und Erklärung zu liefern. Die Mitwirkung der Geführten an der Führung in ihren verschiedenen Facetten spielt dabei eine große Rolle. Partizipation am Führungsprozess findet danach in Form der o.g. Aktivitäten der Geführten (fast) immer statt, auch ohne spezifisches Führungsverhalten oder unterstützende Institutionen. Den Autoren geht es um das Funktionieren von Führung, und nicht um das Ergebnis, auch wenn der Ansatz letztlich impliziert, dass viele den Führungskräften zugeschriebene Ergebnisse und Erfolge eigentlich auf den die Führung initiierenden und lenkenden Aktivitäten der Mitarbeiterinnen und Mitarbeiter beruht.

\subsection{Geteilte Führung}

Das Konzept der geteilten Führung (en. shared leadership) geht im Wesentlichen auf die Arbeiten von Pearce und Sims (2000) sowie Pearce und Conger (Pearce und Conger 2003; Conger und Pearce 2003) zurück. Unter der geteilten Führung wird ein ,dynamischer, interaktiver Einflussprozess zwischen Individuen in Gruppen mit dem Ziel einer wechselseitigen Führung zur Erreichung von Gruppen- oder Organisationszielen oder beiden" verstanden (Pearce und Conger 2003, S. 1). Im Gegensatz zur vertikalen Führung, die auf den formal zugewiesenen Führungspositionen beruht, handelt es sich bei der geteilten Führung um ,eine Ausweitung der Führung auf mehrere oder alle Teammitglieder“ (Hoch und Dulebohn 2017, S. 4). Aus der „Führung eines Teams“ wird eine „Führung durch Team“ (Avolio et al. 2003; Day et al. 2006). Neben der Fokussierung auf Teams wird in den verschiedenen Konzepten zur geteilten Führung die gemeinsame Annahme vertreten, dass dieses Führungsformat sich durch eine laterale Aufteilung der Führungsaufgaben sowie eine gegenseitige Beeinflussung der Beteiligten auszeichnet. Die zu teilenden Führungsrollen sind laut Hiller et al. (2006) Planen und Organisieren, Problemlösung, Unterstützung sowie Entwicklung und Mentoring.

In der Forschung überwiegt die Beschäftigung mit den Voraussetzungen und den Konsequenzen der geteilten Führung. Nach Pearce und Sims (2000, S. 126) hängt das Entstehen und die Ausprägung geteilter Führung von verschiedenen Rahmenbedingungen ab. Die Autoren nennen vor allem Gruppenmerkmale, wie Größe, Diversität, Fähigkeiten und Strategie, oder Aufgabenmerkmale, wie Komplexität, Kreativität und Dringlichkeit der Aufgaben, sowie Merkmale der (Gruppen-)Umwelt, wie kulturelles System, Belohnungssystem und Unterstützungssystem. Des Weiteren wird 
auf die ausgeprägte Selbst-Führung einzelner Teammitglieder hingewiesen, die Führungsteilung ermögliche (z. B. Denis et al. 2012). Eingehend befassen sich die Autorinnen und Autoren auch mit den geteilten mentalen Modellen (en. shared mental models) (Mehra et al. 2006; Carson et al. 2007) als einer der Voraussetzungen für geteilte Führung. Die Entstehung solcher geteilten mentalen Modelle über Führung setzt nach Fletcher und Käufer (2003, S. 38f.) einen Lern- und Entwicklungsprozess im Team voraus, der durch einen generativen Dialog zusammen mit einer hohen Interaktions- und Beziehungsqualität im Team unterstützt wird. Im Idealfall entwickelt das Team ein gemeinsames Verständnis von Zielen und Aufgaben, eine gemeinsame Verantwortung, hohes $\mathrm{Ma}$ an Vertrauen und eine starke Bereitschaft zum Engagement.

In Bezug auf die Konsequenzen der geteilten Führung überwiegt in der entsprechenden Forschung eine starke normative Neigung: Es werden im Wesentlichen positive Folgen der geteilten Führung im Bereich Teamleistung thematisiert. Bereits Pearce und Sims (2000, S. 126) erwarten ausschließlich positive Effekte auf der Gruppenebene wie Effektivität, Gruppenverhalten, (Leistungs-)Potential, Zufriedenheit, Verbundenheit und Zusammenhalt in der Gruppe. Gar vor organisationaler Korruption soll sie schützen (Pearce et al. 2008). Auch Crevani et al. (2007) argumentieren in universalistischer Manier, dass geteilte Führung positive Konsequenzen gleichzeitig auf der individuellen Ebene (z. B. weniger Stress, besserer Ausgleich zwischen dem Beruflichen und Privaten, Werteveränderungen), auf der Ebene der Organisationen (z. B. bessere Akzeptanz der Komplexität, gestiegene Relevanz der Kommunikation, Stabilität der Managementprozesse) wie auch auf der gesellschaftlichen Ebene (z. B. niedrige Machtkonzentration und Machtmissbrauch, höheres Engagement der Minderheiten) nach sich zieht. Diese Tendenz einer dominant positiven Sicht auf das Konzept bleibt auch in den aktuellen Metaanalysen bestehen (z. B. D'Innocenzo et al. 2016; Wang et al. 2014; Wu et al. 2020), die sich hauptsächlich mit der Leistung der Teams mit geteilter Führung auseinandersetzen. Nur einige wenige Autoren, wie z.B. Wegge et al. (2012) merken an, dass geteilte Führung sowohl konstruktive als auch destruktive Formen, wie z.B. aversives Führen, aufweisen kann und diese in der Forschung eine stärkere Beachtung erfahren sollten.

Die aus der geteilten Führung resultierenden Paradoxien in Organisationen hingegen werden in der Forschung nur marginal besprochen. So weisen Fletcher und Käufer (2003) zum einen darauf hin, dass geteilte Führung zu widersprüchlichen Anforderungen für Führungskräfte führen wird, die in den hierarchischen Organisationen sozialisiert sind. Zum anderen geben die Autoren zu bedenken, dass die übliche Managementsprache, die sich aus den jahrhundertelangen Erfahrungen einer hierarchischen Ordnung speist, Verhalten, welches diesen tradierten Vorstellungen nicht entspricht, wie dies der Fall bei der geteilten Führung sein dürfte, auf Kommunikations- und Akzeptanzschwierigkeiten stoßen kann. Zum dritten ist der Konflikt zwischen geteilter Führung und den restlichen Praktiken in Organisationen zu beachten, die meist auf Individuen ausgerichtet sind, wie z.B. Beförderungsentscheidungen. Geteilte Führung würde eine Umstellung auf teambezogenes Personalmanagement erfordern.

Die starke Aufmerksamkeit, die in der Forschung zur geteilten Führung der Teameffektivität gewidmet wird, spricht aus unserer Sicht für eine Effizienzorientierung dieses Konzeptes. Mit geteilter Führung werden derart starke Erwartungen als die Leistungssteigerung geknüpft, dass die Forschungsstudien im Wesentlichen darauf fokussieren, in sorgfältig zusammengestellten Forschungsdesigns Rahmenbedingungen herauszustellen, unter denen die Leistungszuwächse durch geteilte Führung in Teams gesichert werden können. Eine Debatte darüber, inwiefern geteilte Führung auch zu einer faktischen Machtverteilung in Organisationen führt, findet in dieser Forschungsrichtung kaum statt.

\subsection{Verteilte Führung}

Die zentrale Überlegung des Konzeptes der verteilten Führung (en. distributed leadership) ist, dass sich die Führung als Prozess der Einflussnahme verteilt auf verschiedene Formen oder Medien vollzieht (vgl. für einen zusammenfassenden Überblick auch Lang und Rybnikova 2014, S. $158 \mathrm{ff}$., $165 \mathrm{ff}$.).

Gronn (2002), der als einer der ersten Autoren den Begriff verwendet, führt in seiner Konzeption von verteilter Führung neben Aspekten geteilter und spontan entstehender Führungsbeziehungen in Gruppen auch Elemente an, die sich in institutionalisierten Praktiken äußern. In einem späteren Beitrag spricht er von unterschiedlichen Führungskonfigurationen (Gronn 2009). Hoch et al. (2007, S. 51) sprechen vom „simultanen Wirken unterschiedlicher Führungsinstanzen“. Sie beschreiben verteilte Führung unter Ausschluss der Selbstführung als „Muster der Einflussnahme auf Personen in Teams durch gleichgestellte Instanzen der Organisation, der Führung und der Gruppenmitglieder" (Hoch et al. 2007, S. 52). Ähnlich beschreibt Konrath (2014, S. 293) im nah verwandten Konzept der verstreuten Führung (en. dispersed leadership) diese als Einflussnahme durch direkte Führer, Team-Mitglieder, und strukturelle Mechanismen wie organisationale Prozeduren, kulturelle Normen, aufgabenbezogene Strukturen, Informationspolitik usw.

Oborn et al. (2013) gehen noch etwas weiter. Sie entwickeln ein sozio-materielles Modell der verteilten Führung, bei dem Führung als kollektiver Prozess aufgefasst wird, 
der sich über mehrere Gruppen der im Prozess beteiligten Akteurinnen und Akteure sowie den von ihnen angewendeten Instrumenten und Praktiken verteilt. Insbesondere werden auch materielle Artefakte wie Besprechungsräume und Notfallprotokolle hervorgehoben, die eine Verteilung von Führung und damit auch den Grad der Partizipation der verschiedenen Akteurinnen und Akteure am Führungsprozess ermöglichen, verhindern und konfigurieren. Führung wird so stärker in einen weiteren Organisationskontext eingebettet.

Mit Blick auf Partizipationsüberlegungen im Führungsprozess ist zu beachten, dass im Rahmen der verschiedenen konzeptionellen Beiträge und empirischen Studien kein direkter Fokus auf eine Partizipation der Geführten erfolgt. Vielmehr werden verschiedene Formen, Muster oder Konfigurationen beschrieben, in denen Führungsaktivitäten, Verantwortung und Macht verteilt werden. Partizipation kann in diesem Sinne in Gestalt von ermächtigten Stellvertreterinnen und Stellvertretern und Gruppenmitgliedern, mit gemeinsamen Entscheidungsgremien, aber auch in eine weitgehende Beteiligung ermöglichenden Strukturen, Entscheidungsregeln, übergreifenden kulturellen Normen oder Werten (vgl. Bolden 2011, S. 257) gesehen werden.

Dabei geht es beim Konzept der verteilten Führung, vor allem in den Studien im britischen Kontext, eher um eine Beschreibung von Mustern, ihrer Wirkungsweise und entsprechende Rahmenbedingungen für ihre Einführung, z.B. kooperative Arbeitsstrukturen oder eine offene Organisationskultur. Bolden (2011) konstatiert dagegen eine überwiegend normative Orientierung verteilter Führung im US-Kontext, wobei zugleich größere Überschneidungen bei Begrifflichkeiten und Inhalten mit der geteilten Führung (en. shared leadership) erfolgen. Entsprechend finden sich dort auch verstärkte Effizienzerwartungen an eine verteilte/ geteilte Führung.

\section{Fazit: Partizipative Führung zwischen wertebasiertem gesellschaftlichem Führungsideal und effizientem Führungsmuster}

Die obigen Ausführungen machen die Vielfalt von konzeptionellen Überlegungen, die im Bereich partizipativer Führung entwickelt worden sind, deutlich. Eine bereits in die Jahre gekommene Schlussfolgerung erweist sich als immer noch zutreffend: „Partizipative Führung ist ein vages Konzept. Für die einen bedeutet es Entscheidungsfindung in der Gruppe, für die anderen eine gegenseitige Zielsetzung, und für die dritten bedeutet es mehr zuhören und weniger reden“ (Greiner 1973, S. 11).

Ungeachtet der Unterscheide teilen alle genannten Ansätze die kritische Attitüde gegenüber einer auf ausgewählte
Individuen zentrierten Führungssicht. Diese heroisierende Führungslehre, die die Führungskräfte mit elitären Privilegien und Kompetenzen ausstattet, wird als unzeitgemäß abgelehnt. Die stattdessen vorgeschlagene plurale Führungsperspektive fasst Führung als einen Prozess auf, an dem viele oder alle Organisationsmitglieder teilnehmen. Die Führung wird dabei gewissermaßen veralltäglicht.

Die Konzepte ähneln sich jedoch auch in ihren Schwachstellen. So bestehen kaum Bezüge zwischen den Klassikern und den aktuellen Ansätzen, obwohl die Parallelen offensichtlich sind, z.B. zwischen den von Mary Parker Follett und den Ansätzen von Gastil oder Raelin. Auch sind die Konzepte zur partizipativen Führung alle stark auf den unmittelbaren Teamkontext beschränkt. Es fehlen sowohl Bezüge zu industriellen Beziehungen, zur organisationalen Demokratie wie auch zum kulturellen und institutionellen Umfeld, wie z.B. nationalspezifischen Wirtschaftssystemen.

Gleichzeitig wird es klar, dass sich die besprochenen Konzepte in ihrer paradigmatischen Ausrichtung durchaus unterscheiden. Während die einen in der partizipativen Führung ein demokratisches Führungsideal zu erkennen glauben, dass die herrschende Führungslogik herausfordert, wie z. B. Follett, Lewin, Gastil, Raelin, Blom und Alvesson, gilt für die anderen die partizipative Führung als ein weiteres Instrument zur Effizienzsteigerung in Organisationen, wie das Führungskontinuum von Tannenbaum und Schmidt, Vroom und Yetton oder die Konzepte der geteilten Führung. In der aktuellen Führungsforschung dominieren die letzteren (z.B. Drescher und Garbers 2016; Serban und Roberts 2016; Zhu et al. 2018; Pitelis und Wagner 2019). Damit überwiegt die Tendenz zur Instrumentalisierung partizipativer Führung für die Leistungssteigerung in Organisationen. Die demokratieorientierten Konzepte der partizipativen Führung scheinen hingegen deutlich weniger Aufmerksamkeit auf sich zu ziehen.

Im letzten Teil unserer Argumentation gehen wir darauf ein, inwiefern die Debatte um agiles Management die Beachtung der demokratieorientierten Überlegungen zu beleben vermag.

\section{Agiles Management - partizipative Führung im neuen Gewand?}

Auch agiles Management wird gern als eine Ablehnung von hierarchieorientierten Organisationspraktiken hochstilisiert. Es geht auf das sogenannte ,agile Manifest“ zurück, das 2001 von 17 Softwareentwicklern veröffentlicht wurde und ein Grundgerüst für das Agieren in den zunehmend komplexen und chaotischen Systemen bietet. Denkbar schlicht gehalten, enthält das Manifest vier Grundwerte und zwölf Prinzipien, die im Wesentlichen die Kunden- 
nähe, die Zeiteffizienz und die Veränderbarkeit hervorheben. Längst hat die Agilitätsidee die Softwareentwicklungsbranche verlassen und etabliert sich zu einer neuen Managementmode. Führung und Führungsverständnisse bilden hier eine Kerndimension des agilen Managements (z.B. Andresen 2019), nicht zuletzt weil die Selbstorganisation der Teams als eines der zwölf Agilitätsprinzipien formuliert wurde. Zwar verweisen die Autorinnen und Autoren in Zusammenhang mit agiler Führung gern auf ,dienende Führung“" (z. B. Gloger und Rösner 2014, S. 90, Hofert 2018, S. 51), man kann in den Diskussionen aber zahlreiche Anleihen aus den Konzepten zur partizipativen Führung erkennen.

Trotz einer starken Akzentuierung von Selbstorganisationen in Teams wird Führung im Kontext des agilen Managements keinesfalls als redundant angesehen. Umgekehrt, die Relevanz der Führung bleibt erhalten, lediglich die damit zusammenhängenden Aufgaben und die Anforderungen an die Führungskräfte verändern sich stark (Gloger und Rösner 2014, S. 89). Zum einen sei Führung dafür verantwortlich, zusammen mit den Mitarbeitenden Strukturen zu schaffen, die die Selbstorganisation und Eigenverantwortung der Beteiligten unterstützen und den Sinn der Aufgaben zu verdeutlichen (Gloger und Rösner 2014). Zum anderen sorgen agile Führungskräfte für eine Zusammenarbeit innerhalb und zwischen den Teams.

Eine weitere im Diskurs zum agilen Management anzutreffende Idee stellt Führung als entkoppelt von einer Person dar (Hofert 2018, S. 51). Verschiedene Führungsaufgaben können dann auf diverse Rollen innerhalb des Teams verteilt werden, so dass gleichzeitig mehrere Teammitglieder Führungsaufgaben erledigen. Hofert (2018, S. 51) bringt in ihrem Buch ein Beispiel aus dem Kontext des ScrumProgramms an: Während im Scrum dem Product-Owner die Weiterentwicklung des Produkts obliegt, ist der ScrumMaster dafür zuständig, Probleme und Hindernisse aus dem Weg zu räumen. Dabei wird von einer Egalität der Mitglieder ausgegangen: niemand wird über jemand anderen gestellt, ,die Führungskraft ist also nicht Hauptabteilungsleiter, sondern hat die Rolle des Scrum-Masters" (Hofert 2018, S. 51). Die Macht wird nicht aufgrund der hierarchischen Stellung ausgeübt, sondern im Rahmen der momentanen Rolle (Hofert 2018, S. 54), die auch von Teams bestimmt werden kann.

Agile Führung wird auch als letzte Entwicklungsphase der Führungsmodelle angesehen. So präsentiert Hofert (2018) in ihrem Buch ein Kontinuum, das mit der autoritären Führung startet und mit der agilen Führung endet (Hofert 2018, S. 56). In dieser Phase überwiegt „komplette, konsequente Selbstorganisation“. Die Führungskraft füllt ,nicht mehr eine Position gemäß ihrer Persönlichkeit aus, sondern eine Rolle, die vom Unternehmen definiert ist" (Hofert 2018, S. 58).
Betrachtet man die Ausführungen zur agilen Führung aus der Perspektive der hier aufgezeigten Konzeptionen zu partizipativer Führung, stellt man Interessantes fest. Zwar gerieren sich agiles Management und agile Führung als Managementrevolutionen, im konzeptionellen Kern sind es aber klassische Ideen der Führungspartizipation und Selbstorganisation in Teams, die in der traditionellen Managementlehre oft zu kurz kamen und bei denen die Agilitätsmode für einen neuen Aufwind sorgt. Lediglich werden im Agilitätsdiskurs jegliche Verweise auf die klassischen oder auch neuen Konzepte zu partizipativer Führung ausgespart, auch wenn manchen Überlegungen ihre Herkunft direkt anzusehen ist, wie z.B. der agilen Führung als der letzten Phase der Führungsmodelle bei Hofert (2018). Es stellt lediglich eine marginale Umformulierung des Führungskontinuums nach Tannenbaum und Schmidt (1973) dar, wobei die letzten „Phasen“ neuerdings mit der ,agilen Führung" gleichgesetzt werden. Auch das Rollenverständnis der entpersonalisierten agilen Führung nach Hofert (2018) scheint gleichzeitig an die Konzepte zur geteilten Führung oder demokratischer Führung nach Gastil (1994) anzuknüpfen. Nicht nur vermisst man entsprechende Literaturverweise, sondern auch eine Diskussion über die engen Bezüge zwischen der Vergangenheit und der Gegenwart der Managementkonzepte. Bemerkenswert ist auch, welche Ideen der partizipativen Führung Eingang in die Agilitätsdiskussion gefunden haben: Es ist eine recht willkürliche Mischung zwischen den Anleihen aus den demokratieorientierten und den effizienzorientierten Konzepten, ohne dies zu reflektieren oder den jeweiligen konzeptionellen Unterbau zu berücksichtigen. Die Effizienzorientierung im Sinne einer effizienten Befriedigung der Kundenwünsche erfährt im Rahmen der agilen Führung eine leichtfüßige Vermählung mit demokratischer Idee der Machtumverteilung in Scrum-Teams, zumindest auf rhetorischer Ebene. Inwiefern sich diese konzeptionell durchaus bemerkenswerte Verbindung auch in der Praxis bewährt, bleibt eine Frage für zukünftige empirische Betrachtungen.

Danksagung Wir danken den anonymen Gutachterinnen oder Gutachtern für die anregenden, kritischen wie konstruktiven Hinweise zu unserem Manuskript, die - so hoffen wir - zur Präzisierung unserer Argumentation beigetragen haben.

Funding Open Access funding provided by Projekt DEAL.

Open Access Dieser Artikel wird unter der Creative Commons Namensnennung 4.0 International Lizenz veröffentlicht, welche die Nutzung, Vervielfältigung, Bearbeitung, Verbreitung und Wiedergabe in jeglichem Medium und Format erlaubt, sofern Sie den/die ursprünglichen Autor(en) und die Quelle ordnungsgemäß nennen, einen Link zur Creative Commons Lizenz beifügen und angeben, ob Änderungen vorgenommen wurden.

Die in diesem Artikel enthaltenen Bilder und sonstiges Drittmaterial unterliegen ebenfalls der genannten Creative Commons Lizenz, sofern sich aus der Abbildungslegende nichts anderes ergibt. Sofern das be- 
treffende Material nicht unter der genannten Creative Commons Lizenz steht und die betreffende Handlung nicht nach gesetzlichen Vorschriften erlaubt ist, ist für die oben aufgeführten Weiterverwendungen des Materials die Einwilligung des jeweiligen Rechteinhabers einzuholen.

Weitere Details zur Lizenz entnehmen Sie bitte der Lizenzinformation auf http://creativecommons.org/licenses/by/4.0/deed.de.

\section{Literatur}

Alvesson, M., \& Blom, M. (2018). Beyond leadership and followership: working with a variety of modes of organizing. Organizational Dynamics, 48(1), 28-37.

Andresen, J. (2019). Führung - der entscheidende Erfolgsfaktor. In M. Lang \& S. Scherber (Hrsg.), Der Weg zum agilen Unternehmen. Strategien, Potenziale, Lösungen (S. 129-152). München: Hanser.

Avolio, B. J., Sivasubramaniam, N., Murry, W.D., Jung, D., \& Garger, J. W. (2003). Assessing shared leadership. Development and preliminary validation of a team multifactor leadership questionnaire. In C.L. Pearce \& J.A. Conger (Hrsg.), Shared leadership. Reframing the hows and whys of leadership (S. 143-171). Thousand Oaks: SAGE.

Blom, M., \& Alvesson, M. (2014). Leadership on demand: followers as initiators and inhibitors of managerial leadership. Scandinavian Journal of Management, 30, 344-357.

Bolden, R. (2011). Distributed leadership in organizations: a review of theory and research. International Journal of Management Review, 13(3), 251-269.

Braverman, H. (1974). Labour and monopoly capital: the degradation of work in the twentieth century. New York: Monthly Review Press.

Breisig, T. (1990). Skizzen zur historischen Genese betrieblicher Führungs- und Sozialtechniken. Hochschulschriften zum Personalwesen. München/Mering: Hampp.

Carey, A. (1967). The Hawthorne studies: a radical criticism. American Sociological Review, 32(3), 403-416.

Carson, J. B., Tesluk, P.E., \& Marrone, J. A. (2007). Shared leadership in teams: an investigation of antecedent conditions and performance. Academy of Management Journal, 50(5), 1217-1234.

Child, J. (2013). Mary Parker Follett. In M. Witzel \& M. Warner (Hrsg.), The Oxford handbook of management theories (S. 74-93). Oxford: Oxford University Press.

Conger, J. A., \& Pearce, C.L. (2003). A landscape of opportunities: future research on shared leadership. In C. L. Pearce \& C. A. Conger (Hrsg.), Shared leadership. Reframing the hows and whys of leadership (S. 285-303). Thousand Oaks: SAGE.

Crevani, L., Lindgren, M., \& Packendorff, J. (2007). Shared leadership: a post-heroic perspective on leadership as a collective construction. International Journal of Leadership Studies, 3(1), 40-67.

Day, D. V., Gronn, P., \& Salas, E. (2006). Leadership in team-based organizations: on the threshold of a new era. The Leadership Quarterly, 17(3), 211-216.

Denis, J.-L., Langley, A., \& Sergi, V. (2012). Leadership in the plural. The Academy of Management Annals, 6(1), 211-283.

D'Innocenzo, L., Mathieu, J.E., \& Kukenberger, M. R. (2016). A metaanalysis of different forms of shared leadership-team performance relations. Journal of Management, 42(7), 1964-1991.

Drescher, G., \& Garbers, Y. (2016). Shared leadership leadership and commonality: a policy-capturing study. Leadership Quarterly, 27(2), 200-217.

Fiedler, F.E. (1967). A theory of leadership effectiveness. New York: McGraw-Hill.

Fletcher, J. K., \& Käufer, K. (2003). Shared leadership: paradox and possibility. In C.L. Pearce \& C. A. Conger (Hrsg.), Shared lea- dership. Reframing the hows and whys of leadership (S. 21-47). Thousand Oaks: SAGE.

Follett, M.P. (1940a). Power. In H.C. Metcalf \& L. Urwick (Hrsg.), Dynamic administration. Collected papers of Mary Parker Follett (S. 95-116). New York und London: Harper and Brothers.

Follett, M.P. (1940b). The giving of orders. In H. C. Metcalf \& L. Urwick (Hrsg.), Dynamic administration. Collected papers of Mary Parker Follett (S. 50-70). New York und London: Harper and Brothers.

Follett, M. P. (1995). The essentials of leadership. In P. Graham (Hrsg.), Mary Parker Follett - prophet of management. A celebration of writings from the 1920s (S. 163-177). Boston: Harvard Business School Press.

Gastil, J. (1994). A definition and illustration of democratic leadership. Human Relations, 47(8), 953-975.

Gesigora, C. (2015). Mayo, Elton (1933) The human problems of an industrial civilization. New York: Macmillan. In S. Kühl (Hrsg.), Schlüsselwerke der Organisationsforschung (S. 453-457). Wiesbaden: Springer VS.

Gill, R. (2011). Theory and practice of leadership (2. Aufl.). Los Angeles et al.: SAGE.

Gloger, B., \& Rösner, D. (2014). Selbstorganisation braucht Führung. Die einfachen Geheimnisse agilen Managements. München: Hanser.

Greiner, L.E. (1973). What managers think of participative leadership. Harvard Business Review, 51(2), 111-117.

Gronn, P. (2002). Distributed leadership as a unit of analysis. The Leadership Quarterly, 13(4), 423-451.

Gronn, P. (2009). Leadership configurations. Leadership, 5(3), 381-394.

Haslam, S. A., Wegge, J., \& Postmes, T. (2009). Are we on a learning curve or a treadmill? The benefits of participative group goal setting become apparent as tasks become increasingly challenging over time. European Journal of Social Psychology, 39, 430-446.

Hiller, N. J., Day, D. V., \& Vance, R. J. (2006). Collective enactment of leadership roles and team effectiveness: a field study. The Leadership Quarterly, 17(4), 387-397.

Hinrichs, P. (1984). Um die Seele des Arbeiters. Arbeitspsychologie, Industrie- und Betriebssoziologie in Deutschland. Köln: Pahl-Rugenstein.

Hoch, J.E., \& Dulebohn, J.H. (2017). Team personality composition, emergent leadership and shared leadership in virtual teams: a theoretical framework. Human Resource Management Review, 27, 678-693.

Hoch, J.E., Andreßen, P., \& Konradt, U. (2007). E-Leadership und die Bedeutung Verteilter Führung. Wirtschaftspsychologie, 3, 50-58.

Hofert, S. (2018). Agiler Führen: einfache Maßnahmen für bessere Teamarbeit, mehr Leistung und höhere Kreativität (2. Aufl.). Wiesbaden: Springer Gabler.

Holtbrügge, D. (2018). Personalmanagement (7. Aufl.). Wiesbaden: Springer Gabler.

Kieser, A. (2014). Managementlehren - von Regeln guter Praxis über den Taylorismus zur Human Relations-Bewegung. In A. Kieser \& M. Ebert (Hrsg.), Organisationstheorien (7. Aufl. S. 73-117). Stuttgart: Kohlhammer.

Kleingeld, A., van Mierlo, H., \& Arends, L. (2011). The effects of goal setting on group performance: a meta-analysis. Journal of Applied Psychology, 96(6), 1289-1304.

Konrath, U. (2014). Toward a theory of dispersed leadership in teams: model, findings, and directions for future research. Leadership, 10(3), 289-307.

Lang, R. (2014). Participative leadership in cross-cultural leadership research: a misconception? In O. Kranz \& T. Steger (Hrsg.), Zwischen Instrumentalisierung und Bedeutungslosigkeit. Mitarbeiter-Partizipation im organisationalen Kontext in Mittel- und Osteuropa (S. 77-92). München/Mering: Hampp.

Lang, R., \& Rybnikova, I. (2014). Verteilte und geteilte Führung: Alle machen mit? In R. Lang \& I. Rybnikova (Hrsg.), Aktuelle Füh- 
rungstheorien und -konzepte (S. 151-179). Wiesbaden: Springer Gabler.

Lewin, K., Lippit, R., \& White, R. K. (1939). Patterns of agressive behavior and experimentally created "social climates". Journal of Social Psychology, 10, 271-299.

McLarney, C., \& Rhyno, S. (1999). Mary Parker Follett: visionary leadership and strategic management. Women in Management Review, 14(7), 292-304.

Mehra, A., Smith, B. R., Dixon, A. L., \& Robertson, B. (2006). Distributed leadership in teams: the network of leadership perceptions and team performance. Leadership in team-based organizations. The Leadership Quarterly, 17(3), 232-245.

Mendenhall, M.E., \& Marsh, W. J. (2010). Voices from the past: Mary Parker Follet and Joseph Smith on collaborative leadership. Journal of Management Inquiry, 19(4), 284-303.

Neuberger, O. (2002). Führen und führen lassen. Stuttgart: UTB.

Northouse, P.G. (2019). Leadership: theory and practice (8. Aufl.). Thousand Oaks et al.: SAGE.

Oborn, E., Barrett, M., \& Dawson, S. (2013). Distributed leadership in policy formulation: a sociomaterial perspective. Organization Studies, 34(2), 253-276.

Pearce, C. L., \& Conger, J. A. (2003). All those years ago: the historical underpinnings of shared leadership. In C. L. Pearce \& J. A. Conger (Hrsg.), Shared leadership. Reframing the hows and whys of leadership (S. 1-18). Thousand Oaks: SAGE.

Pearce, C. L., \& Sims, H. P. (2000). Shared leadership: toward a multilevel theory of eadership. Advances in Interdisciplinary Studies of Work Teams, 7, 115-139.

Pearce, C.L., Manz, C.C., \& Sims, H.P. (2008). The roles of vertical and shared leadership in the enactment of executive corruption: implications for research and practice. The Leadership Quarterly, 19(3), 353-359.

Pitelis, C. M., \& Wagner, J.D. (2019). Strategic shared leadership and organizational dynamic capabilities. Leadership Quarterly, 30(2), 233-242.

Raelin, J. (2006). Does action learning promote collaborative leadership? Academy of Management Learning \& Education, 5(2), 152-168.

Raelin, J. A. (2014). Imagine there are no leaders: reframing leadership as collaborative agency. Leadership, 12(2), 131-158.

Roethlisberger, F. J., \& Dickson, W. J. (1939). Management and the worker: an account of a research program conducted by the Western Electric Company, Hawthorne works, Chicago. Cambridge: Harvard University Press.

Seidel, E. (1978). Betriebliche Führungsformen. Stuttgart: Poeschel.

Serban, A., \& Roberts, A. J. B. (2016). Exploring antecedents and outcomes of shared leadership in a creative context: a mixed-methods approach. Leadership Quarterly, 27(2), 181-199.

Steffensen, B. (2015). Roethlisberger, Fritz Jules, Dickson, William J. (with assistance and collaboration of Harold A. Wright) (1939): Management and the worker. In S. Kühl (Hrsg.), Schlüsselwerke der Organisationsforschung (S. 453-457). Wiesbaden: Springer VS. An account of a Research Program conducted by the Western Electric Company, Hawthorne Works, Chicago. Cambridge: Harvard University.

Tannenbaum, R., \& Schmidt, W.H. (1973). How to choose a leadership pattern. Harvard Business Review, 51, 3-12.

Vroom, V.H., \& Jago, A.G. (1978). On the validity of the VroomYetton model. Journal of Applied Psychology, 63, 151-162.

Vroom, V.H., \& Yetton, P.W. (1973). Leadership and Decision Making. London/Pittsburgh: University of Pittsburgh Press.

Wang, D., Waldman, D. A., \& Zhang, Z. (2014). A meta-analysis of shared leadership and team effectiveness. Journal of Applied Psychology, 99(2), 181-198.

Weber, W. G., Unterrainer, C. \& Höge, T. (2019). Psychological research on organisational democracy: A meta-analysis of individual, organisational, and societal Outcomes. Applied Psychology: An International Review (early online view)
Wegge, J., \& Haslam, S.A. (2005). Improving work motivation and performance in brainstorming groups: effects of three group goal setting strategies. European Journal of Work and Organizational Psychology, 14(4), 400-430.

Wegge, J., Jeppesen, H.-J., \& Weber, W.G. (2012). Broadening our perspectives: we-leadership is both less romantic and more democratic. Industrial and Organizational Psychology, 5, 418-420.

Wegge, J., Jeppesen, H.-J., Weber, W. G., Pearce, C. L., Silvia, S. A., Pundt, A., Jonsson, T., Wolf, S., Wassenaar, C. L., Unterrainer, C., \& Piecha, A. (2010). Promoting work motivation in organizations: Should employee involvement in organizational leadership become a new tool in the organizational psychologists' kit. Journal of Personnel Psychology, 9, 154-171.

Weibler, J. (2016). Personalführung (3. Aufl.). München: Vahlen.

Wu, Q., Cormican, K., \& Chen, G. (2020). A meta-analysis of shared leadership: antecedents, consequences, and moderators. Journal of Leadership \& Organizational Studies, 27(1), 49-64.

Wunderer, R. (1995). Kooperative Führung. In A. Kieser, G. Reber \& R. Wunderer (Hrsg.), Handwörterbuch der Führung (S. 1369-1386). Stuttgart: Schäffer-Poeschel.

Wunderer, R., \& Grunwald, W. (1980). Kooperative Führung. Führungslehre, Bd. II. Berlin/New York: De Gruyter.

Yukl, G. (2006). Leadership in organizations (6. Aufl.). Upper Saddle River: SAGE.

Yukl, G. (2013). Leadership in organizations (8. Aufl.). Upper Saddle River: SAGE.

Zhu, J., Liao, Z., Yam, K. C., \& Johnson, R.E. (2018). Shared leadership: a state-of-the-art review and future research agenda. Journal of Organizational Behavior, 39(7), 834-852.

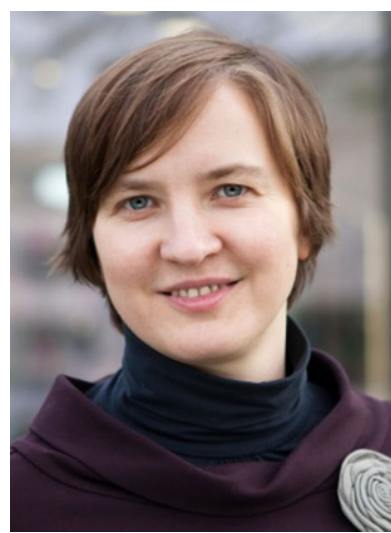

Irma Rybnikova ist Professorin für Personalmanagement und Organisation an der Hochschule HammLippstadt. In ihrer Forschung befasst sie sich mit den konzeptionellen und praktischen Fragen der Mitarbeiterführung, Mitarbeiterpartizipation und der Diversität in Organisationen.

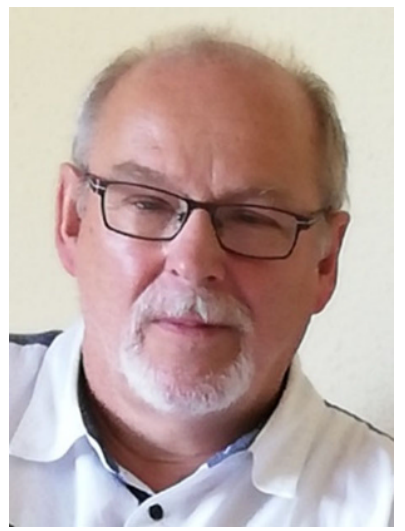

Rainhart Lang war bis 2017 als Professor für Organisation und Arbeitswissenschaften an der TU Chemnitz tätig. Seit seiner Emeritierung lehrt er weiterhin in Bachelor- und Masterstudiengängen dieser Universität und ist auch als Gastprofessor an der Universität Regensburg tätig. In seiner Forschung befasst er sich unter anderem mit Führung im interkulturellen Kontext sowie der Professionalisierung im Personalmanagement. 